Si mul t aneous $i$ on and neut $r$ al evapor at $i$ on in aqueous nanodrops: Experi ment, theory, and nol ecul ar dynamics si mul at i ons

\begin{tabular}{|l|l|}
\hline 著者 & $\begin{array}{l}\text { H gashi Hi denor i, Tokum Takuya, Hogan } \\
\text { Chr i st opher J. Jr. , Suda Hor oshi, Set o } \\
\text { Takaf um, Ot ani Yoshi o }\end{array}$ \\
\hline $\begin{array}{l}\text { j our nal or } \\
\text { publ i cat i on ti tl e }\end{array}$ & Physi cal Chemi st ry Chem cal Physi cs \\
\hline vol une & 17 \\
\hline nunber & 24 \\
\hline page range & $15746-15755$ \\
\hline year & $2015-06-28$ \\
\hline URL & ht t p: //hdl . handl e. net /2297/48651 \\
\hline
\end{tabular}




\section{Simultaneous Ion and Neutral Evaporation in Aqueous Nanodrops: Experiment, Theory, and Molecular Dynamics Simulation}

Hidenori Higashi ${ }^{1 *}$, Takuya Tokumi ${ }^{1}$, Christopher J. Hogan Jr. ${ }^{2}$, Hiroshi Suda ${ }^{3}$, Takafumi Seto ${ }^{1}$, Yoshio Otani ${ }^{1}$

${ }^{1}$ Faculty of Natural Systems, Institute of Science and Engineering, Kanazawa University, Kanazawa, JAPAN

${ }^{2}$ Department of Mechanical Engineering, University of Minnesota, Minneapolis, MN, USA

${ }^{3}$ Home Appliances Development Center, Corporate Engineering Division, Appliances Company, Panasonic Corporation, Kusatsu, JAPAN

Submitted to:

Phys Chem Chem Phys

*To whom correspondence should be addressed: hhigashi@mail.kanazawa-u.ac.jp 
We use a combination of tandem ion mobility spectrometry (IMS-IMS, with differential mobility analyzers), molecular dynamics (MD) simulations, and analytical models to examine both neutral solvent $\left(\mathrm{H}_{2} \mathrm{O}\right)$ and ion (solvated $\mathrm{Na}^{+}$) evaporation from aqueous sodium chloride nanodrops. For experiments, nanodrops were produced via electrospray ionization (ESI) of an aqueous sodium chloride solution. Two nanodrops were examined in MD simulations: a 2,500 water molecule nanodrop with $68 \mathrm{Na}^{+}$and $60 \mathrm{Cl}^{-}$ions (an initial net charge of $z=+8$ ), and (2) a 1,000 water molecule nanodrop with $65 \mathrm{Na}^{+}$and $60 \mathrm{Cl}^{-}$ions (an initial net charge of $z=+5$ ). Specifically, we used MD simulations to examine the validity of a model for the neutral evaporation rate incorporating both the Kelvin (surface curvature) and Thomson (electrostatic) influences, while both MD simulations and experimental measurements were compared to predictions of the ion evaporation rate equation of Labowsky et al [Anal Chim Acta, 2000, 406, 105-118]. To within a single fit parameter, we find excellent agreement between simulated and modeled neutral evaporation rates for nanodrops with solute volume fractions below 0.30 . Similarly, MD simulation inferred ion evaporation rates are in excellent agreement with predictions based on the Labowsky et al equation. Measurements of the sizes and charge states of ESI generated $\mathrm{NaCl}$ clusters suggest that the charge states of these clusters are governed by ion evaporation, however, ion evaporation appears to have occurred with lower activation energies in experiments than was anticipated based on analytical calculations as well as MD simulations. Several possible reasons for this discrepancy are discussed. 


\section{INTRODUCTION}

Ion evaporation (ion emission) is a process in which bare or solvated ions are emitted from highly charged drops, driven by sufficiently high electrostatic potential at the drop surface ${ }^{1-}$

4. This phenomenon is frequently encountered with nanodrops resulting from electrospray ionization (ESI), and a large body of evidence suggests that in ESI, ions deriving from most small molecules ( $\sim$ sub kiloDalton) are generated through the ion evaporation process ${ }^{5-7}$. Conversely, while compact, macromolecular ions (e.g. multiply charged protein ions electrosprayed from non-denaturing solutions) appear to be produced by the charge residue mechanism $^{8}$ (in which they simply retain the charge of their former nanodrop, upon solvent evaporation), there is evidence that it is ion evaporation that governs their eventual charge distribution ${ }^{9-12}$. Therefore, despite prior ambiguity in the mechanism by which ESI leads to singly charged small molecule ions and multiply charged macromolecular ions, it appears now, that with the exception of highly linear macromolecules (which are likely ionized via chain ejection from nanodrops ${ }^{13-16}$ ), ion evaporation influences both small molecule ion yield as well as macromolecular charge distributions ${ }^{13}$.

There is hence need to quantify ion evaporation rates from nanodrops, such that small molecule ion yields and macromolecular ion charge distributions can be predicted in ESI. The earliest studies of ion evaporation were performed by Thomson \& Iribarne $^{1}$, who both demonstrated that it is the ion evaporation process which leads to gas phase ion production and developed a model linking the electrostatic potential energy of a drop to the ion evaporation rate. Seminal studies were later performed by Fernandez de la Mora and colleagues, first experimentally (with ion mobility spectrometry) examining the validity of the rate form of Thomson \& Iribarne $e^{4}$, second developing an improved ion evaporation rate equation ${ }^{17}$ and 
showing good agreement between its predictions and measurements ${ }^{2}, 18$, and additionally demonstrating that ion evaporation can occur not only from nanodrops but from the tips of electrospray Taylor cones themselves ${ }^{3,19}$. Distinct from these experimental and theoretical efforts, a number of researchers, beginning with Znamenskiy et $\mathrm{a}^{20}$ and including the works of Consta and colleagues ${ }^{21-23}$ as well as Konermann and colleagues ${ }^{24-26}$, have used Molecular Dynamic (MD) simulations to directly observed, computationally, ions evaporating from nanodrops. Notable amongst these prior works, Ichiki \& Consta $^{23}$ computed the approximate radii of evaporating, solvated chloride ions from water drops in simulations, finding good agreement with model predictions of Labowsky et al ${ }^{17}$.

However, what remains to be demonstrated is a clear intercomparison of measurements, theoretical predictions, and MD simulations of ion evaporation for identical systems. Such comparison is necessary to better verify the proposed rate equations for ion evaporation. Therefore, in this study, we perform tandem ion mobility spectrometry (IMS-IMS) measurements of electrospray generated $\mathrm{NaCl}$ cluster ions generated via aqueous ESI, and compare the inferred charge distributions of these clusters to both theoretical predictions as well as to MD simulations of the same system. Importantly, we show that in comparing experiments, models, and computations, not only is it important to correctly model ion evaporation, but also neutral (solvent) evaporation from nanodrops, as ion evaporation and neutral evaporation occur simultaneously. In the sections that follow we describe the IMS-IMS experiments and MD simulations performed, and present models of neutral and ion evaporation, based on transition regime theory for aerosols ${ }^{27,28}$ and the model of Labowsky et a $1^{17}$, respectively. 


\section{EXPERIMENTAL \& COMPUTATIONAL METHODS}

\section{IMS-IMS Measurements}

A major hindrance in examining nanodrop dynamics is that typically, when evaporating, the lifetimes of nanodrops are exceedingly small relative to the timescales of applicable experimental systems. For this reason, it is difficult to observe ion evaporation directly. Instead, common practice is measurement of the size and charge (two dimensional size and charge distribution) of residue cluster ions ${ }^{4,5,18}$, nanoparticles $^{29-31}$, or macromolecules ${ }^{8,11,12}$ generated via ESI; these species persist as multiply charged entities in the gas phase after nanodrop evaporation. In linking the size and charge distribution of these species to nanodrop ion evaporation kinetics the assumption is invoked that the residue entities (aerosol particles) were ionized via the charge residue mechanism ${ }^{8}$ and bear the charge of the nanodrop at the moment solvent evaporation completed. Following prior studies, we also make this assumption here, and used IMS-IMS to infer the size and charge distributions of ESI-generated sodium chloride $(\mathrm{NaCl})$ clusters in the $3-10 \mathrm{~nm}$ size range for comparison to both analytical models and MD simulations. The experimental system employed is depicted in Figure 1. In it, a $\sim 8.5 \mathrm{mM}$ aqueous solution of $\mathrm{NaCl}$ was electrosprayed using a stainless steel capillary (Hamilton, model 7747-02, inner diameter $=110 \mu \mathrm{m}$, outer diameter $=240 \mu \mathrm{m})$ connected to a syringe pump (Harvard Apparatus model 70-2205). The solution flowrate was $1.67 \mu \mathrm{L} \mathrm{min}$ ma $^{-1}$ and a voltage of $\sim 2.5 \mathrm{kV}$ was applied to the metal capillary (relative to the surrounding grounded chamber) to facilitate formation of a stable Taylor cone. Taylor cone stability was observed visually during all experiments using a CCD camera (Sony model XC-75). The electrospray produced droplets in a chamber where $1.51 \mathrm{~min}^{-1}$ of particle-free, dry air was used to entrain droplets. Based on electrospray drop diameter scaling laws ${ }^{32}$, we estimate that the original droplets produced were 
close to 1 micrometer in diameter; however, rapid solvent evaporation and Coulombic fissions ${ }^{33}$ led to the production of nanodrops with sufficiently high surface electrostatic energies to promote ion evaporation. Nanodrops themselves evaporated rapidly as they were transported out of the electrospray chamber, leaving multiply charged sodium chloride cluster ions.

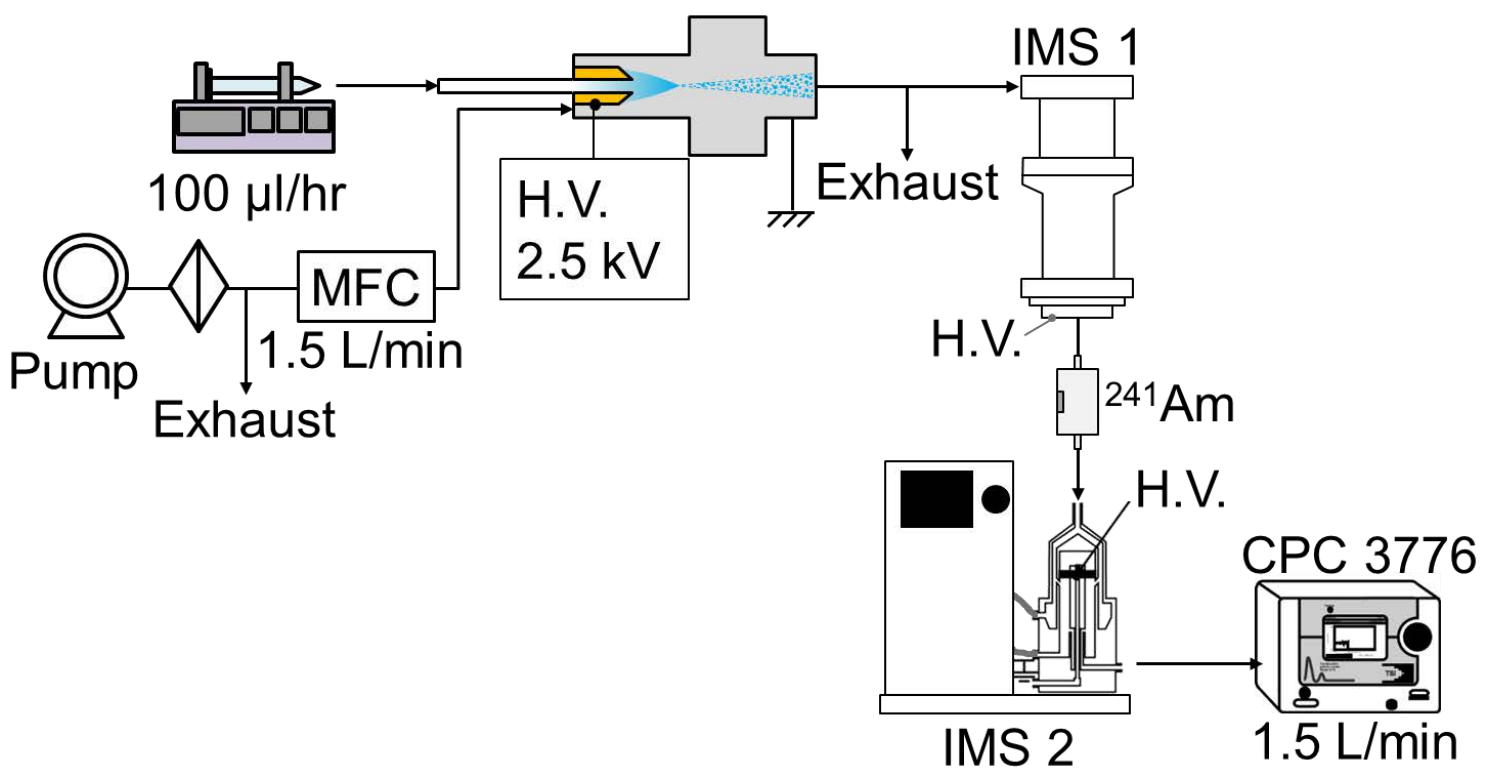

Figure 1. A schematic of the IMS-IMS system employed in measuring the size and charge distributions of ESI generated $\mathrm{NaCl}$ clusters. MFC- Mass flow controller; H.V.- High Voltage; IMS-1: A Vienna-type DMA, IMS-2: A TSI Nano-DMA (model 3085). CPC 3776: A condensation particle counter).

The cluster ions were then passed into a differential mobility analyzer (DMA, a type of IMS instrument ${ }^{34,35}$ ), which was a Vienna-type model ${ }^{36}$ designed for sub $100 \mathrm{~nm}$ diameter particles. This DMA was operated with a recirculating sheath flowrate of $151 \mathrm{~min}^{-1}$ of air and all $1.51 \mathrm{~min}^{-1}$ of the cluster ion flow was sampled into the DMA. DMAs are ion mobility filters ${ }^{34}$, capable of isolating particles/ions of a specific ion mobility, $Z_{p}$. The first DMA was operated with a constant voltage difference $(0.1-2.5 \mathrm{~V})$ between electrodes to transmit only cluster ions with mobility within $+/-10 \%$ (based on the DMA resolving power) of a prescribed value. This 
small voltage was well below the applied voltages commonly used in DMA analysis; for this purpose a high accuracy voltage source (Keithley Corporation, model 2410) was utilized. Approximately monomobile cluster ions exited the $1^{\text {st }}$ DMA, though with a distribution of sizes and charge states. These cluster ions were then passed into a chamber containing an Am-241 source $(100 \mu \mathrm{Ci})$; in this chamber the Am-241 source generated $\alpha$-irradiation, which in turn produced roughly equal concentrations of positive and negative ions (from impurities deriving from system components with non-zero vapor pressure ${ }^{37}$ ). Via recombination, primarily with negative ions, the clusters were brought close to a steady-state charge distribution ${ }^{37,38}$, wherein the cluster charge distribution was solely a function of cluster size and independent of initial charge state. Prior studies, both experimental ${ }^{39}$ and theoretical ${ }^{37}$, show clearly that in the sub 10 $\mathrm{nm}$ size range (of interest here), at steady-state and for ions generated in air at room temperature, most clusters are neutral, and those that are charged are only singly charged (i.e. the charge state, $\mathrm{z}= \pm 1$ ). Subsequent to the Am-241 source chamber, clusters were passed through a second DMA (a TSI model 3085 nano-DMA) which was coupled with a single cluster-sensitive ultrafine condensation particle counter (CPC, TSI model 3776). These two instruments were operated in tandem as a scanning mobility particles spectrometer $\left(\mathrm{SMPS}^{40}\right)$; because clusters were singly charged after the Am-241 chamber, those transmitted through the second DMA were of known charge state, hence measurement of their mobility in the second DMA enabled inference of their size. In total, by stepping the first DMA through different voltages, and for each first DMA applied voltage using the SMPS for size distribution measurements, the IMS-IMS combination employed enabled inference of combined cluster size and charge distributions. The link between measured mobilities and inferred sizes and charges described in the "Data Processing and Analysis," sub-section of the "Results \& Discussion" section. 


\section{Molecular Dynamics Simulations}

MD simulations of the evaporation of aqueous sodium chloride nanodrops were performed using Materials Explorer 5.0 (Fujitsu Co. Ltd.). Specifically, in simulations, two nanodrops were examined, mimicking those observed in IMS-IMS experiments; (1) a 2,500 water molecule nanodrop with $68 \mathrm{Na}^{+}$and $60 \mathrm{Cl}^{-}$ions (an initial net charge of $\mathrm{z}=+8$ ), and (2) a 1,000 water molecule nanodrop with $65 \mathrm{Na}^{+}$and $60 \mathrm{Cl}^{-}$ions (an initial net charge of $\mathrm{z}=+5$ ). Described in greater detail in the supplemental information, water molecules were modeled using the SPC/E rigid molecular model ${ }^{41}$ considering Lenard-Jones (12-6) and Coulomb potentials with partial charges on water molecules. $\mathrm{Na}^{+}$and $\mathrm{Cl}^{-}$were similarly modeled with Lenard-Jones (12-6) and Coulomb potentials (integer charge states) considered with the Lenard-Jones parameters used provided in the supplemental information. Evaporation of both solvent and ions was modeled at a fixed temperature of $460 \mathrm{~K}$ within a periodic boundary condition cubic domain which was $90 \dot{A}$ per side. The periodic condition was employed to enable considering of long range nanodrop-nanodrop interactions; rarely are nanodrops isolated from one another and in ESI they are known to interact Coulombically ${ }^{42}$. Prior to monitoring evaporation, water molecules, as well as $\mathrm{Na}^{+}$and $\mathrm{Cl}^{-}$ions, were placed in a smaller cubical domain with dimensions chosen to give a density of $1 \mathrm{~g} \mathrm{~cm}^{-3}$ (for the entire system). The resulting configuration was allowed to "equilibrate" at $300 \mathrm{~K}$ for $1 \mathrm{ps}$. The domain was then expanded to its full size $(90 \dot{A})$ and simulations were continued for an additional $10 \mathrm{ps}$, resulting in the formation of roughly spherical nanodrops (without evaporation observed). Following this, the temperature was increased to $460 \mathrm{~K}$ and evaporation, both of solvent and of ions, was monitored by computing radial distribution functions for water, $\mathrm{Na}^{+}$, and $\mathrm{Cl}^{-}$, as functions of simulation time. From these 
distribution functions a nanodrop diameter was defined, as described in the "Data Processing and Analysis" sub-section. Constant temperature was maintained in simulations by shifting molecule and ion speed linearly every 100 timesteps (as well as when the temperature was increased) such that the translational kinetic energy was roughly constant in simulations. Though the evaporation of water molecules from the nanodrop leads to cooling, in our simulations the additional re-condensation of water compensates for this influence, and the constant temperature approximation has only a minor influence on results (we note that comparison to analytical models, presented subsequently, we also assume nanodrops are isothermal). Further, as our simulations target the final stages of evaporation, the temperature decrease on the timescale of the simulation is negligibly small. In addition, though periodic boundaries were employed on the domain surface, when a sodium collided the domain boundary, water molecules within $\sim 30 \dot{A}$ of the domain were removed. While this introduced some ambiguity into the background water vapor pressure in simulations, this was necessary to prevent ion-induced water molecule clustering (i.e. ion-induced nucleation ${ }^{43}$ ) and the formation of nanodrops elsewhere in the system. 


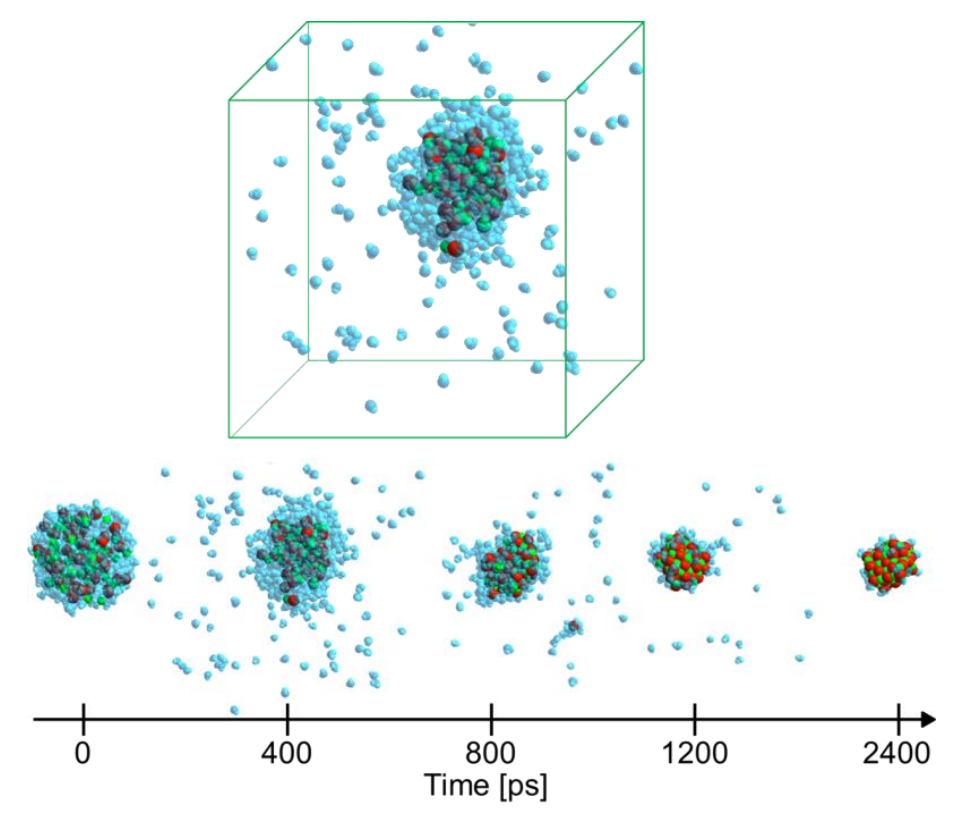

Figure 2. A depiction of an evaporating nanodrop in simulations. The upper panel shows a nanodrop with the rough domain bounds depicted, while the lower time series displays snapshots of the nanodrop at selected simulation times (every $400 \mathrm{ps}$ ).

\section{RESULTS \& DISCUSSION}

To compare IMS-IMS results and MD simulation results, appropriate data analysis methods are necessary. We describe the approach taken in data analysis in the subsequent section, followed by description of analytical models of neutral and ion evaporation, for additional comparison to experiments and computations. Following these two sections, we divide the discussion of results into two components: (1) discussion of the observed neutral evaporation rate in MD simulations (we note this is not observable in IMS-IMS experiments) and that predicted analytically, and (2) an intercomparison of measurements of the charge and size distributions of nanoclusters, simulated ion evaporation from nanodrops, and analytical models of simultaneous neutral and ion evaporation. Readers not concerned with the details of data analysis and analytical model develop may forego the immediate two sub-sections in lieu of the final two sub-sections. 


\section{Data Processing and Analysis}

To infer size and charge distributions from IMS-IMS measurements, we first note that for sub $10 \mathrm{~nm}$ clusters in air near $300 \mathrm{~K}$ and atmospheric, the Knudsen number, i.e. the ratio of the gas mean free path $(\sim 67 \mathrm{~nm})$ to cluster radius, is sufficiently large to approximate cluster drag as free molecular. This enables use of the Mason-Schamp equation ${ }^{44}$ to link the inferred mobility $\left(Z_{c}\right)$ of a cluster ion to its collision cross section $(\Omega)$. For the first DMA employed, $\left(Z_{c, 1}\right)$, the Mason-Schamp equation can be written as:

$$
Z_{c, 1}=\sqrt{\frac{\pi m_{\text {gas }}}{8 k T}} \frac{3 z e}{4 \rho_{\text {gas }}} \frac{1}{\Omega_{z}}
$$

where $z$ is the original cluster charge state (of interest), $m_{\text {gas }}$ is the molecular of weight of air, $k$ is Boltzmann's constant, $T$ is the gas temperature, $\rho_{\text {gas }}$ is the gas mass density, and $e$ is the unit electron charge. As made evident in equation (1a), the collision cross section $\left(\Omega_{z}\right)$ is a function of the charge state, in large part due to the polarization potential between air molecules and multiply charged cluster ions ${ }^{45}$. In contrast, after charge reduction, the Mason-Schamp equation for clusters transmitted through second DMA $\left(Z_{c, 2}\right)$ is:

$$
Z_{c, 2}=\sqrt{\frac{\pi m_{\text {gas }}}{8 k T}} \frac{3 e}{4 \rho_{\text {gas }}} \frac{1}{\Omega_{0}}
$$

where $\Omega_{0}$ is the baseline collision cross section for the cluster ion, i.e. the collision cross section in the absence of the ion induced dipole potential. Through mobility measurement in the second DMA, $\Omega_{0}$ can be directly calculated, as the charge state is known. A cluster diameter $\left(d_{c}\right)$ can then be inferred by approximating clusters as spheres, and employing the equation ${ }^{46}$ :

$$
\Omega_{0}=\frac{\pi}{4} \xi\left(d_{c}+d_{g}\right)^{2}
$$


where $d_{g}$ is the effective gas molecule diameter, taken to be equivalent to $0.3 \mathrm{~nm}^{46}$, and $\xi$ is the momentum scattering factor, approximately equivalent to 1.36 (deriving from the measurements of Millikan ${ }^{47,48}$ ) in the size range of interest here. Following Larriba-Andaluz $\&$ coworkers ${ }^{49-51}$ as well as others ${ }^{45,52}$, the spherical assumption additionally leads to a link between $\Omega_{\mathrm{z}}$ and $\Omega_{0}$ via the equation:

$$
\frac{\Omega_{z}}{\Omega_{0}} \approx L\left(\Psi_{p o l}\right)
$$

where $L\left(\Psi_{\text {pol }}\right)$ is a dimensionless polarization correction factor, dependent on the polarization energy to thermal energy ratio $\left(\Psi_{\mathrm{pol}}\right)$ approximately as:

$$
\begin{aligned}
& L\left(\Psi_{p o l}\right) \approx 1+\Psi_{p o l}\left(0.322+\frac{1}{\xi}\left(0.0625+0.1212 \Psi_{p o l}\right)\right) \text { for } \Psi_{\mathrm{pol}}<1 \\
& \Psi_{p o l}=\frac{2 \alpha_{p o l} Z^{2} e^{2}}{\pi\left(d_{c}+d_{g}\right)^{2} k T}
\end{aligned}
$$

where $\alpha_{\mathrm{pol}}$ is the polarizability of the gas molecules (approximately $1.7 \times 10^{-30} \mathrm{~m}^{3}$ for molecular nitrogen and oxygen). For larger values of $\Psi_{p o l}$ (much greater than unity), and for cluster ions significantly more massive than the background gas, the function $L$ collapses to a form which leads to ion mobilities which are functions of neither the charge state nor the cluster diameter (the polarization limit) ${ }^{53}$. Combining equations (1a-d) leads to the relationship:

$$
\frac{Z_{c, 1}}{Z_{c, 2}}=\frac{z}{L\left(\Psi_{p o l}\right)}
$$

With $d_{c}$ inferred via equation (1c) from second DMA measurements, equation (1g) enables evaluation of $z$ from IMS-IMS measurements (it is the only remaining unknown value in this equation). We employed this approach for all CPC-detected particles in IMS-IMS experiments, transforming measured signal intensity (expressed in terms of the size distribution function $\mathrm{dn} / \mathrm{d}\left(\log \left[\mathrm{d}_{\mathrm{c}}\right]\right)$ and corrected for by accounting for the fraction of neutral particles remaining after 
the Am-241 source ${ }^{54}$ ) as a function of $Z_{c, 1}$ and $Z_{c, 2}$ into measured signal intensity as a function of $\mathrm{d}_{\mathrm{c}}$ and $\mathrm{z}$, facilitating comparison to both MD simulations and analytical models. In many instances, we note that non-integer charge levels are inferred; though these values are unphysical and simply arise because of finite instrument resolution, non-integer inferred values do not appreciably influence the comparison of experimental results to MD simulations and analytical models.

To extract quantitative information from MD simulations, approximately every 100 picoseconds we calculated the radial distribution function for all atoms within the system ${ }^{55}$, with respect to the center of mass of the system. Example distributions are provided in the supplemental information. Radial distribution functions were integrated from the outer bounds of the domain to the center of mass, and the nanodrop diameter at each time was inferred from the radial distance (from the center of mass) corresponding to half (50\%) the atoms in the system. Subsequently, a charge state at each time was evaluated by determining the number of sodium and chloride ions within the bounds of the nanodrop. Though this approach would incorrectly estimate the charge level of highly skewed objects, we observed extremely low aspect, highly isotropic nanodrops in all simulations. With this approach, MD simulations led to $d_{c}$ and $z$ as functions of simulation time.

\section{Models of Neutral \& Ion Evaporation}

As noted in the "Introduction" section, to our knowledge analytical models of neutral evaporation and ion evaporation have not been compared simultaneously to both experimental data and MD simulations performed for the same system, and for this reason we attempt this intercomparison here. Two such comparisons are possible: first, as MD simulations yield a 
nanodrop diameter as a function of time, these results can be compared to an appropriate model of neutral evaporation from a highly charged nanodrop. Second, both experiments and simulations yield $z$ as a function of $d_{z}$ (which is time independent) and be compared to coupled models of neutral and ion evaporation.

In comparison of neutral evaporation rates (quantified by the change in nanodrop diameter over time) inferred from MD simulations to models, we remark that MD simulations do not mimic evaporation in vacuo; because of the periodic boundary conditions water nanodrops are evaporating into a background gas of water vapor. Further, the water vapor concentration is not negligibly small, i.e. finite diffusive Knudsen number $\left(\mathrm{Kn}_{\mathrm{D}}\right)^{56,57 /}$ mass transfer transition regime influences on the evaporation rate must be considered. In this regard, the simulations performed here are unique from prior efforts ${ }^{23,26}$ in modeling neutral and ion evaporation from nanodrops. Under arbitrary background gas conditions, the change nanodrop diameter (denoted also as $d_{c}$ as later comparison to the cluster diameters) with time is given by the equation:

$$
\frac{d d_{c}}{d t}=\frac{2 D_{w w} m_{w}}{\rho_{d} k T d_{c}}\left(P_{w, \infty}-P_{d}\right) \frac{1+K n_{D}}{1+1.71 K n_{D}+1.333 K n_{D}^{2}}
$$

where $D_{w w}$ is the water vapor self-diffusion coefficient, $\mathrm{m}_{\mathrm{w}}$ is the water molecular mass, $\rho_{\mathrm{d}}$ is the nanodrop density ( 879.57 at $460 \mathrm{~K}$, used calculations here), $P_{\mathrm{w}, \infty}$ is the far-field water vapor pressure, $P_{d}$ is the nanodrop surface vapor pressure, and $K n_{D}$ is given by the equation ${ }^{58}$ :

$$
K n_{D}=\sqrt{\frac{\pi m_{w}}{2 k T}} \frac{D_{w w}}{d_{c}+d_{w}}
$$

where $d_{w}$ is an effective water molecule diameter (approximated as $0.316 \mathrm{~nm}$ for the SPC/E rigid model). Equations (2a) and (2b) follow from the work of Fuchs \& Sutugin ${ }^{27}$, who used Sahni's solution ${ }^{59}$ to the Boltzmann equation for non-continuum mass transfer of a point mass to a sphere to infer vapor molecule condensation and evaporation rates to particles. More recently, this 
equation has been found to agree well with alternative calculation approaches ${ }^{28}$, as well as experimental data ${ }^{60}$. For the remaining parameters, we invoked the Kelvin-Thomson model ${ }^{43,61}$ to describe the nandrop surface vapor pressure, $P_{d}$ :

$$
P_{d}=P_{s a t} \exp \left(\frac{4 \gamma m_{w}}{\rho_{d} k T d_{c}}-\frac{m_{w} z^{2} e^{2}}{2 \pi^{2} \rho_{d} k T d_{c}^{4}}\left(1-\frac{1}{\varepsilon_{w}}\right)\right)
$$

where $P_{\text {sat }}$ is the water vapor pressure $(1.170 \mathrm{MPa}$ at $460 \mathrm{~K}), \gamma$ is the liquid water surface tension $\left(0.041 \mathrm{~N} \mathrm{~m}^{-1}\right.$ at $\left.460 \mathrm{~K}, \gamma(T)=0.2358(1-T / 647.096)^{1.256\{1-0.625(1-T / 647.096)\}}\right)$ and $\varepsilon_{w}$ is the water dielectric constant (78). The self-diffusion coefficient of water was calculated using ChapmanEnskog theory ${ }^{62}$ :

$$
D_{w w}=\frac{A_{1} T^{3 / 2} m_{w}}{2 \sigma_{w w}^{2} \Omega^{*}} \frac{P_{r e f}}{P_{w, \infty}}
$$

where $\mathrm{A}_{1}=1.12 \times 10^{17} \mathrm{~m}^{4} \mathrm{~s}^{-1} \mathrm{~kg}^{-1} \mathrm{~K}^{-3 / 2}, P_{r e f}=101300 \mathrm{~Pa}($ atmospheric pressure at $300 \mathrm{~K}), \sigma_{w w}^{2}=$ $0.07 \mathrm{~nm}^{2}$ (the effective cross section for water-water collisions), and $\Omega^{*}$ is the dimensionless function $^{63}$ :

$$
\begin{aligned}
& \Omega^{*} \approx \frac{1.06036}{T_{e f f}^{0.1561}}+\frac{0.193}{\exp \left(0.47635 T_{e f f}\right)}+\frac{1.03587}{\exp \left(1.52996 T_{e f f}\right)}+\frac{1.76474}{\exp \left(3.89411 T_{e f f}\right)} \\
& T_{e f f}=\frac{k T}{\mathrm{E}_{w}}
\end{aligned}
$$

$E_{w}$ is the potential well depth for water $\left(1.12 \times 10^{-20} \mathrm{~J}\right)$. The only remaining parameter in the evaporation model (in equations $2 \mathrm{a}$ and $2 \mathrm{~d}$ ) is the effective, far-field water vapor pressure $\left(\mathrm{P}_{\mathrm{w}, \infty}\right)$. Regrettably, because of the need to purge water molecules periodically from systems, this parameter is neither simple to estimate (because of the finite simulation domain) nor a constant in simulations. Therefore, in comparing MD simulation results to models for evaporation, we fit a functional form for $\mathrm{P}_{\mathrm{w}, \infty}$; though this introduces a degree of ambiguity in comparison, shown 
subsequently, excellent agreement is found with a very simple fit function for the far-field vapor pressure, which is applied to both simulation results.

We invoke the model of Labowsky et $\mathrm{al}^{17}$ to predict the rate of ion evaporation. In this model, the rate of change of a nanodrop's charge state $(d z / d t)$ is given by the equation:

$$
\frac{d z}{d t}=-z \frac{k T}{h} \exp \left(-\frac{\Delta}{k T}\right)
$$

where $h$ is Planck's constant, and $\Delta$, the activation energy for ion evaporation, is given by the equation:

$$
\begin{aligned}
& \Delta=\Delta G_{s}-\frac{e^{2}}{2 \pi \varepsilon_{0} d_{c}}\left(F(z)+\alpha_{I E}\right) \\
& F(z) \approx z^{\frac{1}{2}}+0.375 z^{-0.39}-0.875
\end{aligned}
$$

Equation (3c) is a fit to the tabulated information for the function $F(z)$ provided by GameroCastano \& Fernandez de la Mora ${ }^{2} . \Delta G_{s}$ is the solvation energy for $\mathrm{Na}^{+}$ions in water $\left(3.912 \times 10^{-}\right.$ $\left.{ }^{19} \mathrm{~J}\right)^{17} \alpha_{I E}$, the ion evaporation curvature correction factor, typically takes values in the range 0.2-0.7 2, ${ }^{18}$, and only weakly influenced ion evaporation kinetics. Nonetheless, we use experimental data to estimate it, making plots of the minimum cluster diameter observed for a given charge level as a function of $\mathrm{F}(\mathrm{z})$; linearly regression fits to such plots yield the value of $\alpha_{I E}$. Simultaneous solutions of equations (2) and (3) enable us to monitor $d_{c}$ and $z$ as functions of time, for direct comparison to simulations and experiments. In solving equation (3), we again permit non-integer charge values and finding that this has a negligible influence on results. Further, we note that equations (2) and (3) are coupled; z directly influences the surface vapor pressure of a nanodrop (the Thomson effect), while the activation energy for ion evaporation is strongly dependent on $d_{c}$. 


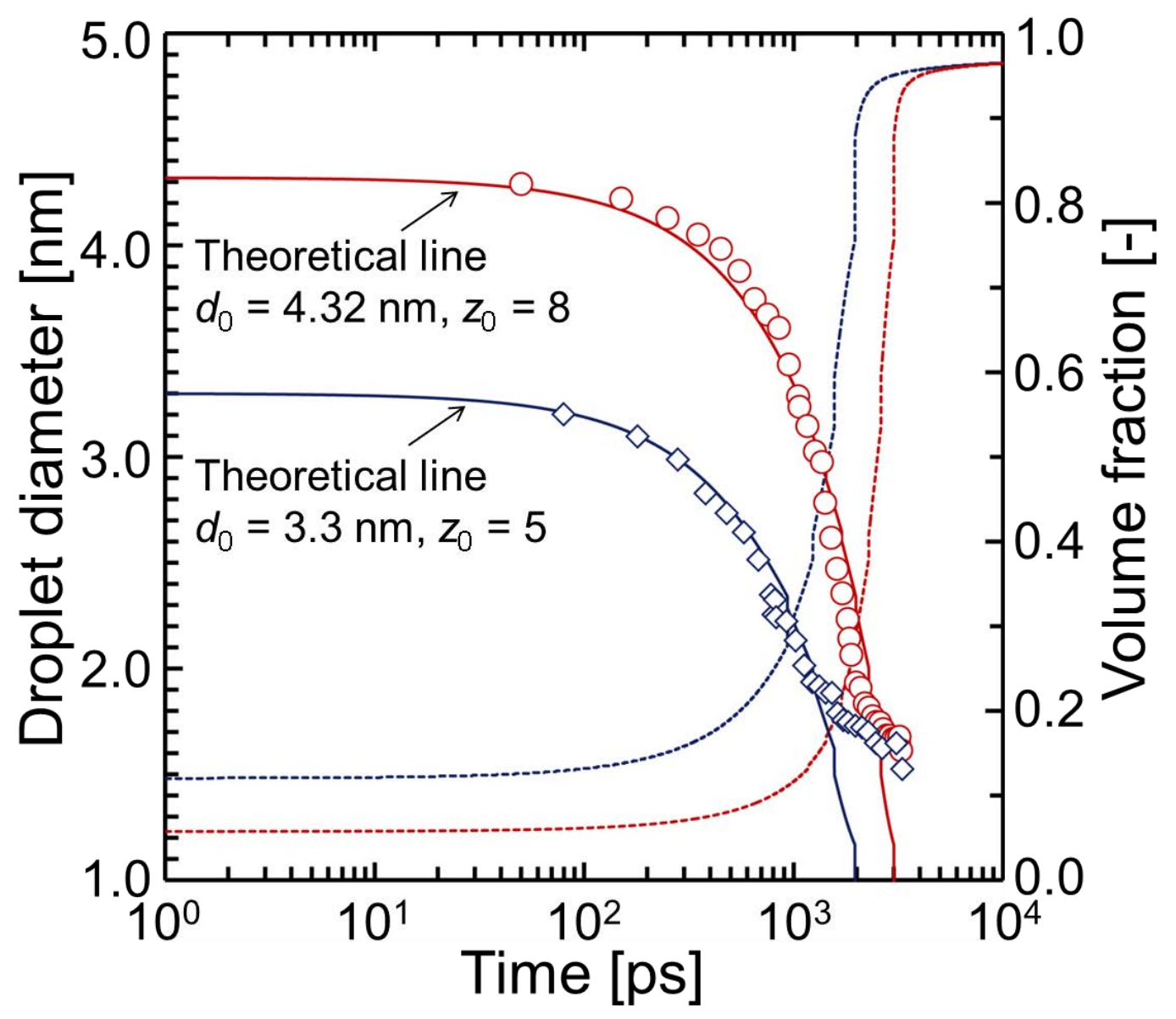

Figure 3. A plot of the radial distribution function drop diameters (in nanometers) as function of simulation time for the two examined nanodrops. $d_{0}$ and $z_{0}$ denote the initial drop diameter and charge state. Symbols denote MD simulation results, while solid lines denote analytical model predictions with the fit relationship $P_{\mathrm{w}, \infty}=0.85 P_{\mathrm{d}}$ under all circumstances. Dashed lines denote the effective sodium chloride volume fraction in drops as a function of time (right vertical axis).

The evaporation rate for solvent is quantified in MD simulations and in modeling via plots of the nanodrop diameter as a function of time (we again remark information on the neutral evaporation rate is not extractible in experiments). For both simulated nanodrops, which had radial distribution function defined diameters of $4.32 \mathrm{~nm}$ (for 2500 water molecules with $\mathrm{z}=+8$ ) and $3.30 \mathrm{~nm}$ (for 1000 water molecules with $\mathrm{z}=+5$ ), respectively, and using equations (2) and (3) with the noted initial $d_{c}$ and $z$ values, such plots are shown in Figure 3. The simulated nanodrop 
diameters (3.30 and $4.32 \mathrm{~nm})$ were slightly smaller than those predicted using the density of pure water as $998 \mathrm{~kg} \mathrm{~m}^{-3}$ (3.95 and $\left.5.23 \mathrm{~nm}\right)$. This difference may be caused from our definition of drop diameter (based upon the radial distribution function), which also appears to influence subsequent comparison to experiments. The large partial charges on water molecules, originating from the SPC/E model may also have some influences on the drop size. A video simulation of the MD simulation for the initially $3.30 \mathrm{~nm}$ nanodrop is provided in the supplemental information. In it, both neutral evaporation and ion evaporation of solvated $\mathrm{Na}^{+}$ ions are clearly evident, hence simulations are immediately found to be in qualitative agreement with expected charged nanodrop behavior. Excellent quantitative agreement is also observed in Figure 3 between MD simulation results and model predictions, with the assumption that $P_{\mathrm{w}, \infty}=$ $0.85 P_{\mathrm{d}}$ under all conditions as the only fit parameter in model predictions. Despite the use of this fit function, such a functional form for $\mathrm{P}_{\mathrm{w}, \infty}$ is reasonable for the simulations as performed (i.e. the vapor pressure is below that of the drop surface and increasing with increasing evaporation), and the remarkably good agreement between simulated and modeled nanodrop evaporation supports the validity of the analytical model invoked in modeling neutral evaporation; thus, results support the validity of the Fuchs-Sutugin mass transfer transition regime rate (and similar functions), as well as the Kelvin-Thomson relation. This finding is further in agreement with recent simulations ${ }^{64}$ which show that the Kelvin relation holds valid for neutral water nanodrops with sizes in the nanometer range. However, earlier MD simulations ${ }^{65}$ of neutral nanodrops show deviation from the Kelvin effect for nanodrops several nanometers in diameter.

Clear deviation between simulated evaporation and modeled is evident for both nanodrops after approximately 1000 ps. In both cases, deviation coincides with the solid volume fraction in nanodrops (i.e. the volume occupied by $\mathrm{Na}^{+}$and $\mathrm{Cl}^{-}$, also plotted in Figure 3 as a 
function of time) increasing to levels beyond $\sim 0.3$. In these circumstances the nanodrop vapor pressure is undoubtedly modified from equation (2c) predictions, as it is known that high concentration salt solution have vapor pressures well below the vapor pressures of pure solvents $^{66}$. To further understand the influence of salt ions on neutral evaporation, we also performed evaporation simulations with nanodrops containing a limited number $\mathrm{Na}^{+}$ions $(0,3$, 5 and 7 sodium ions, respectively, with results shown in the supplemental information). The calculated evaporation rates of nanodrops with these limited number of cations (and no anions) were only slightly higher than those obtained for nanodrops containing higher salt concentrations, until the high salt concentration nanodrops attained higher solid volume fraction. However, we did find that low salt concentration nanodrops adopted highly aspherical shapes after appreciable evaporation, hence it was not possible to use the approach taken here to reliably estimate the drop diameter in these circumstances. 
Ion Evaporation: Model, Experiment, and MD Simulation Comparison
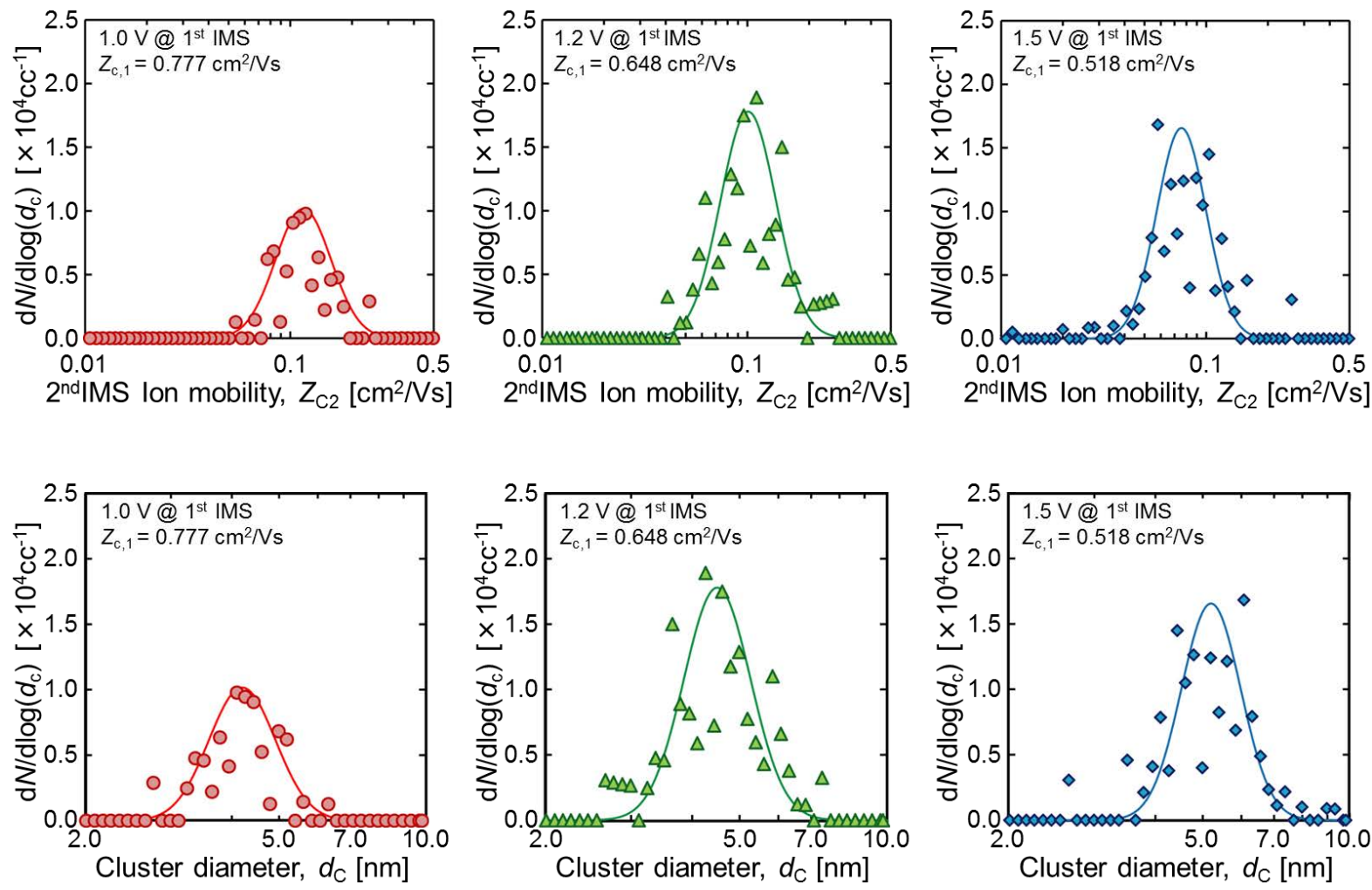

Figure 4. The inferred size distribution functions (in terms of the number concentration per unit $\log$ cluster diameter, obtained directly from the TSI SMPS software), as a function of inferred mobility (upper) and inferred cluster diameter (lower) from the second DMA. Results are shown for selected voltage settings in the first DMA. Symbols correspond to measured signal, with fit Gaussian curves also provided for guidance.

Figure 4 displays raw data of the relative signal intensity measured by the CPC as a function of $Z_{c, 2}$ (upper) and inferred $d_{c}$ (lower) for selected values of $Z_{c, 1}$. Evident is the polydispersity in cluster ion mobility and diameter in the electrospray process as well as also that clusters of a given diameter had variable charge states, and hence variable mobilities resulting from the electrospray process. Using the noted data analysis approach, $z$ values were inferred for all detected clusters. Results were then rounded to the nearest integer charge state, and for each charge state, size distributions were plotted and are shown in the supplemental information (detected signal as a function of cluster diameter were noted). Multiple "local maximum" peaks 
were evident in these distributions, for each, the smallest peak with a $d n / d \log \left(d_{c}\right)$ value exceeding $5000 \mathrm{~cm}^{-3}$ was identified as the minimum sized $\left(d_{\min }\right)$ clusters for the charge state in question. Linear regression to a plot of $d_{\min }$ as a function of $\mathrm{F}(\mathrm{z})$, as is shown in Figure 5, yields $\alpha_{\mathrm{IE}}{ }^{18}$ :

$$
d_{\text {min }}(z)=2 A_{2}\left(F(z)+\alpha_{I E}\right)
$$

where $2 \mathrm{~A}_{2}$ is the slope of the regression curve. Through this approach we find $\alpha_{\mathrm{IE}}=0.391$, in line with prior studies where $\alpha_{\text {IE }}$ is inferred ${ }^{2,18}$.

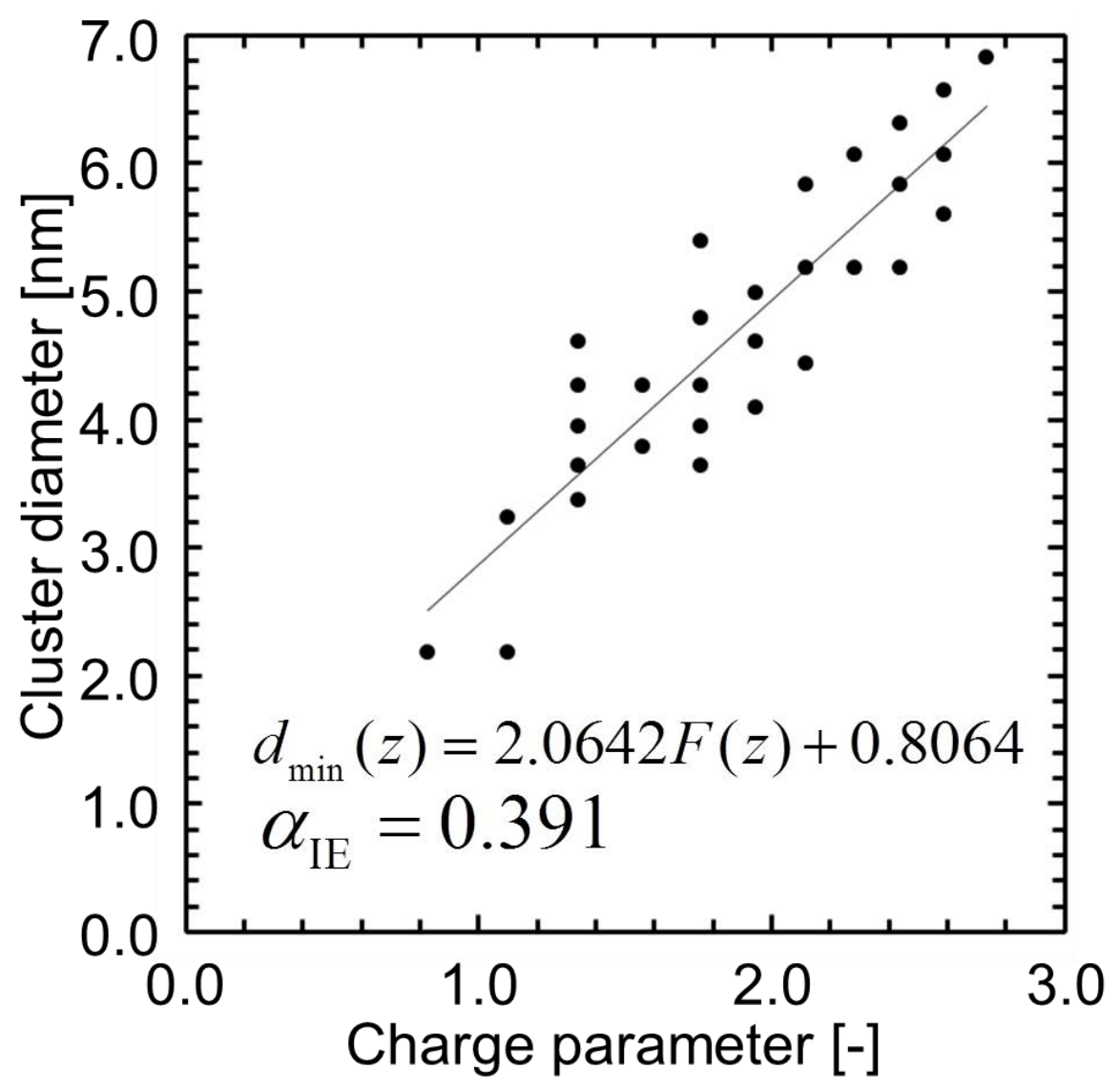

Figure 5. A plot of the minimum diameter, $d_{\min }$, for each charge state, as a function of the charge parameter, $F(z)$. 


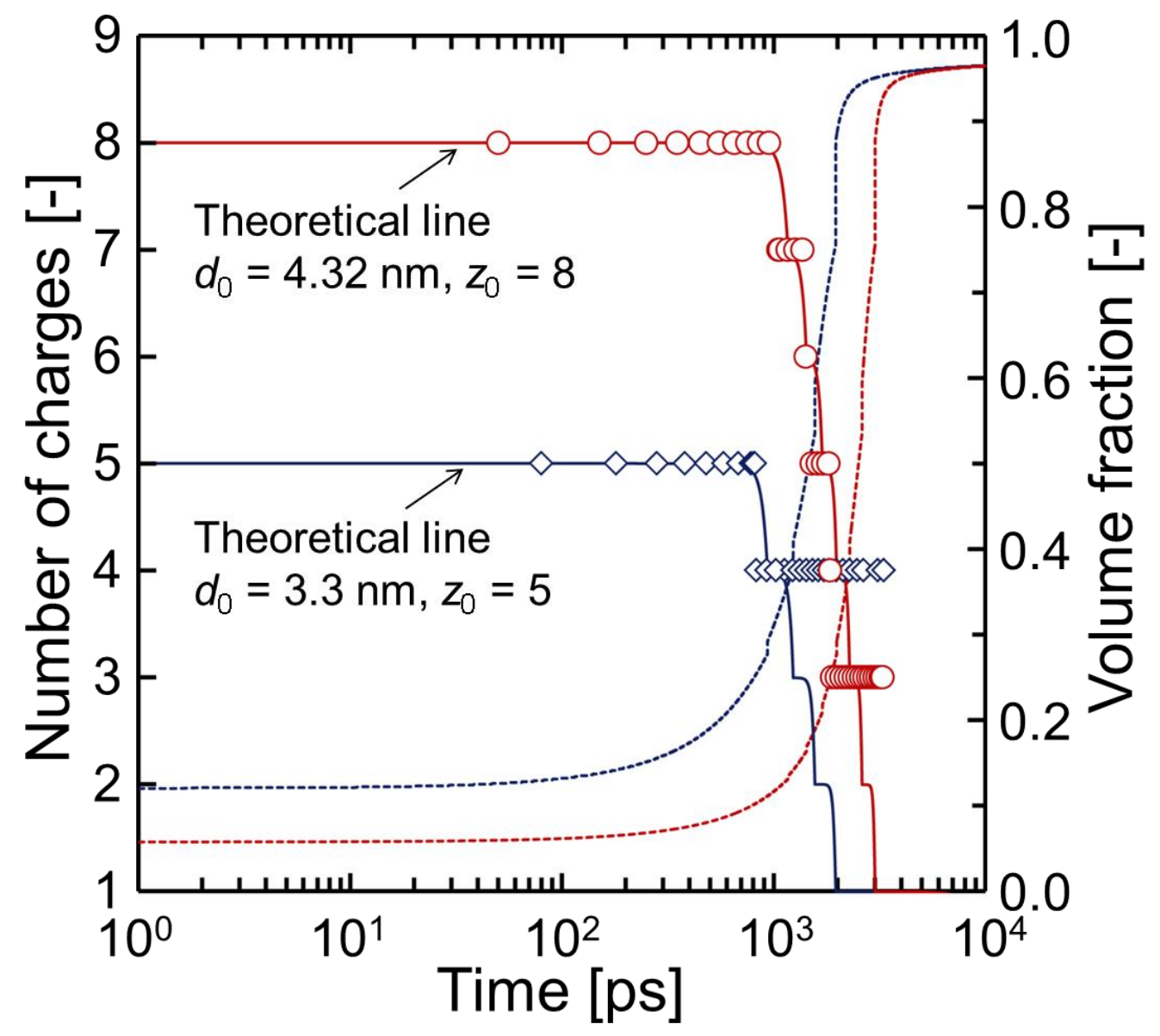

Figure 6. A plot of the charge state as function of simulation time for the two examined nanodrops. $d_{0}$ and $z_{0}$ denote the initial drop diameter and charge state. Symbols denote MD simulation results, while solid lines denote analytical model predictions. Dashed lines denote the effective sodium chloride volume fraction in drops as a function of time (right vertical axis).

The inferred $\alpha_{\text {IE }}$ value was subsequently input into equation (3b) for analytical model predictions. In parallel with Figure 3, Figure 6 is a plot of the charge state $\mathrm{z}$ of nanodrops in both MD simulations and from the analytical model. As with neutral evaporation, excellent agreement between simulations and model predictions is observed when the solid volume fraction of drops is below $\sim 0.3$. Beyond this value, ion evaporation appears to be quenched in MD simulations; this is presumably because of a change in the solvation energy for $\mathrm{Na}^{+}$ions for nanodrops which have appreciably high solute concentrations. Not only do simulations support 
the use of the model of Labowsky et $\mathrm{a}^{17}$ at low solute volume fractions with the parameters employed here (none of which are fit to model results, with the exception of the far-field vapor pressure), they also support the use of experiments where residue cluster size and charge distributions are used to infer ion evaporation kinetics, as simulations suggest that once large fractions of solvent evaporation, the rate of ion evaporation decreases substantially.

Though not apparent in figure 6 , in-line with prior studies ${ }^{5,10}$, when the charge state from both MD simulations and the analytical model is plotted as a function of $d_{z}$, a specific curve arises which is independent of initial conditions after the first ion evaporation event and prior to reaching high solute volume fractions. These curves are overlaid on a contour plot of measured salt cluster signal intensity as a function of $z$ and $d_{\mathrm{c}}$ in Figure 7. In analyzing both measurements and in analytical calculations, we did not round results to the nearest integer charge state. Clearly evident is the strong agreement between MD simulation and analytical model results for $d_{\mathrm{c}}$ as a function $z$. This functional relationship is found to be relatively independent of the input function for the far-field vapor pressure in the analytical model; the $z$ versus drop diameter relationship is more dependent upon the activation energy of ion evaporation; therefore, the agreement observed is further evidence that the model of Labowsky et al explains well ion evaporation.

Though difficult to distinguish in contour plots, particles with charge states ranging from 2-12 were detectable in measurements. Evident in Figure 7 is that experimental observed cluster charge states are lower (for the same diameter) than anticipated based on MD simulations or the analytical model. Even so, experimental measurements are consistent with ion evaporation controlling the charge state on clusters. Additionally overlaid in Figure 7 are the $z$ versus $d_{\mathrm{c}}$ relationships corresponding to constant surface electric field strength of $2.0 \mathrm{~V} \mathrm{~nm}{ }^{-1}$ and $1.4 \mathrm{~V}$ 
$\mathrm{nm}^{-1}$, respectively. Experimental results are bounded by these two curves. Ion evaporation is expected to lead to a nearly size independent surface electric field strength for drops ${ }^{4,9,12}$, which is not expected for drops close to their Rayleigh limit (drops near their Rayleigh limit additionally attain much higher charge levels, as shown by the orange dashed line). Interestingly, the Rayleigh limit and the simulated and theoretical calculation results are somewhat coincident. However, in simulations, we find that charge is ejected from drops in the form of individual cations with only several water molecules bound, which is not consistent with the anticipated outcome of a Rayleigh fission (i.e. multiply charged nanodrops are expected to be ejected during Rayleigh fission ${ }^{33}$. 


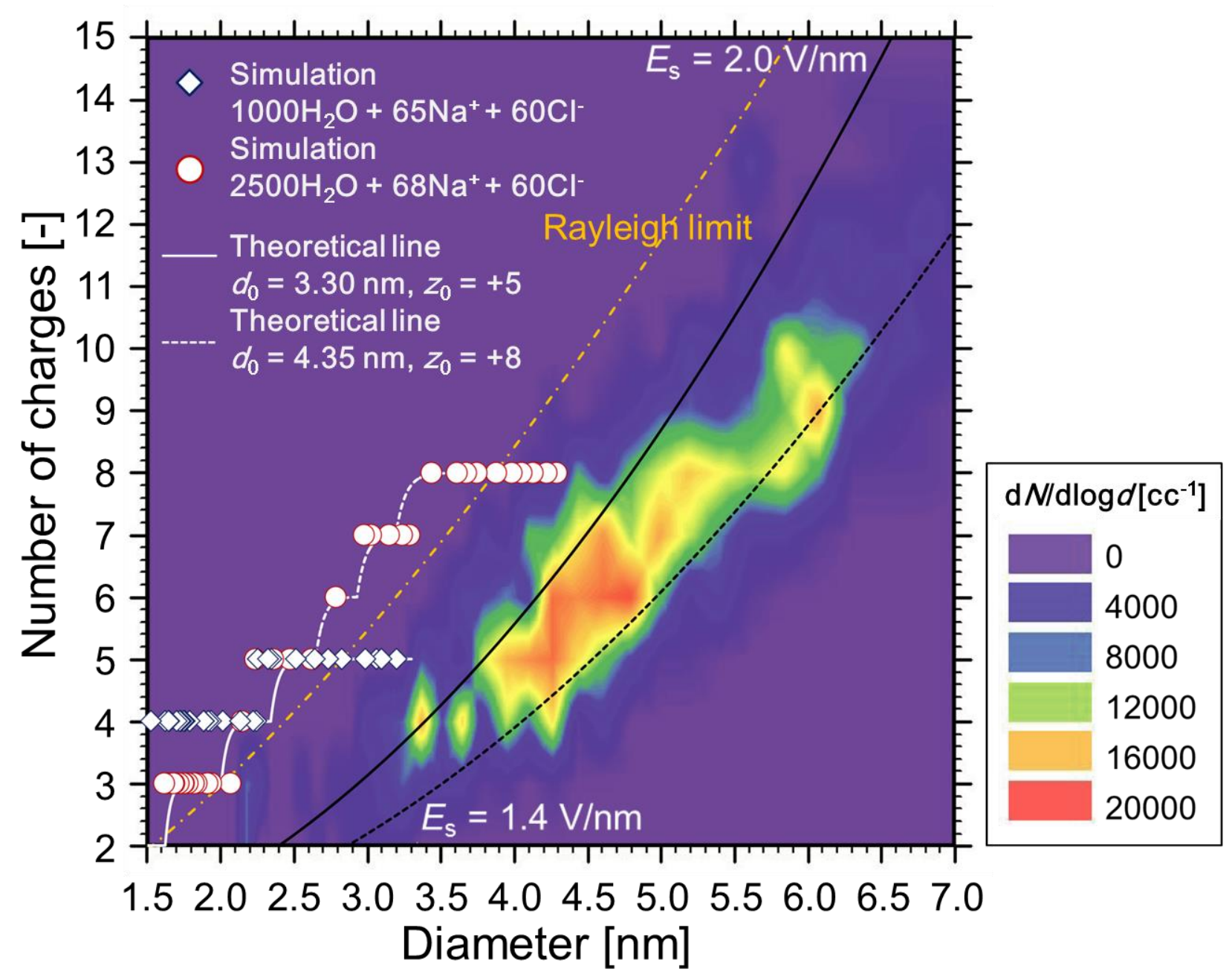

Figure 7. A plot of the charge state as a function of diameter from the analytical model (white lines), MD simulations (symbols), measurements (contour plot denoting size distribution function intensity), and expected for constant electric field strength models as well as at the Rayleigh limit for water.

While in total, both simulations and experiments provide strong evidence for ion evaporation controlling cluster charge states and both permit quantitation of the ion evaporation rate through the relationship between $z$ and diameter, the differences between simulations and experiments merit further discussion. There are several plausible reasons for the observed deviation. First, ion evaporation from solid surfaces is possible ${ }^{2,5,51}$, though simulations suggest 
that the rate of ion evaporation from bare salt cluster surfaces is low when compared to the ion evaporation from water drops. Second, diameter is inferred in different manners in simulations and in measurements; in simulations diameter is selected based on a radial distribution function, while in mobility measurements diameter is inferred from a model linking the mobility (i.e. the inverse of the drag force on a cluster) to an effective diameter. While some ambiguities in this model do arise for salt clusters with nanometer dimensions ${ }^{51}$, they would lead to an ambiguity in cluster diameter of $15 \%$ at most, and thus are also not likely to be the main source of deviation. We do remark that the deviations between experiments and simulations almost completely vanish if the nanodrop diameters are based upon an $80-90 \%$ cutoff criterion in the cumulative radial distribution functions, which is most probably the main source of the discrepancy. This points to a need for more systematic methods of evaluating evaporating nanodrop sizes in MD simulations in future work. Other possible origins of deviation include the fact that higher mobility clusters are transmitted less efficiently in atmospheric pressure IMS systems, and may have slightly contributed to lower observed experimental charge states. However, we find experimental errors in mobility measurement to be unlikely as differential mobility analyzers with known sheath flowrates are straightforward to calibrate. Finally, as ion evaporation kinetics are dependent upon the solvation energy of the evaporating ions, lower solubility impurities present in solutions could ultimately lead to reduced charge levels observed in experiments. We find this to be the most likely source of deviation besides discrepancies in the definition of diameter in simulations and experiments; solute concentrations increase in drops due to neutral evaporation, such that impurities originally in trace amounts may have been present in high enough concentrations to affect ion evaporation kinetics. Overall, we find that the compounding influences of these four phenomena could lead to the observed deviation 
between experiments and simulations, and note further work will be needed to resolve differences in experiments and simulations.

\section{CONCLUSIONS}

We study ion evaporation from nanodrops generated by ESI of aqueous sodium chloride solutions, uniquely comparing experimental measurements to both MD simulation and analytical model results. Two specific comparisons are carried out; neutral evaporation rates are inferred from both MD simulations and models, and ion evaporation kinetics are examined with experiments, simulations, and models. Based on this work, we make the following conclusions:

1. To a single fit parameter, predictions of the neutral evaporation rate for nanodrops considering the Kelvin (curvature influences) and Thomson (electrostatic influences) effects are in good agreement with MD simulations until non-volatile solutes occupy a significant fraction of the drop volume. To our knowledge, such simulations of neutral evaporation from multiply charged nanodrops have not been compared to KelvinThomson based evaporation models previously.

2. The ion evaporation model of Labowsky et $\mathrm{al}^{17}$ is in remarkably good agreement with the results of MD simulations. Combined with the results are prior work ${ }^{2,11,18}$, we find there is strong support for the continued use of this model in describing the rate of ion evaporation from nanodrops.

3. IMS-IMS measurements of the charge distribution on residue sodium chloride clusters reveal lower charge states (for a given cluster size) than are anticipated based on both MD simulations and analytical models, with the discrepancy possibly brought about by compounding effects. Nonetheless, measurements do suggest that the charge level 
attained by clusters is controlled by the evaporation of ions from nanodrops prior to the cessation of neutral solvent evaporation. We suggest that future studies of ion evaporation should focus on improved quantitative prediction of the charge distributions observed on clusters, macromolecules, and nanoparticles aerosolized and ionized via ESI.

\section{SUPPLEMENTAL INFORMATION}

Details on the parameters used in MD simulations, example radial distribution functions, evaporation results for nanodrops without excess sodium chloride, example experimentally inferred size distribution functions at discrete charge states (with a description of $d_{\min }$ determination), and a video of simultaneous neutral and ion evaporation in MD simulations are available online.

\section{ACKNOWLEDGEMENTS}

The authors would like to acknowledge supports and valuable discussions with Dr. Tetsuya Maekawa, Mr. Takeshi Imai and Mr. Jupei Oe at Home Appliances Development Center, Corporate Engineering Division, Appliances Company, Panasonic Corporation.

\section{REFERENCES}

1. B. A. Thomson and J. V. Iribarne, Journal of Chemical Physics, 1979, 71, 4451-4463.

2. M. Gamero-Castano and J. Fernandez de la Mora, J Mass Spectrom, 2000, 35, 790-803.

3. M. Gamero-Castano and J. Fernandez de la Mora, Journal of Chemical Physics, 2000, 113, 815832.

4. I. G. Loscertales and J. Fernandez de la Mora, Journal of Chemical Physics, 1995, 103, 50415060 .

5. M. Gamero-Castano and J. Fernandez de la Mora, Anal Chim Acta, 2000, 406, 67-91.

6. $\quad$ N. B. Cech and C. G. Enke, Analytical Chemistry, 2001, 73, 4632-4639. 
7. S. Nguyen and J. B. Fenn, Proceedings of the National Academy of Sciences, 2007, 104, 11111117.

8. J. Fernandez de la Mora, Anal Chim Acta, 2000, 406, 93-104.

9. C. J. Hogan, J. A. Carroll, H. W. Rohrs, P. Biswas and M. L. Gross, Journal of the American Chemical Society, 2008, 130, 6926-6927.

10. C. J. Hogan, J. A. Carroll, H. W. Rohrs, P. Biswas and M. L. Gross, Analytical Chemistry, 2009, 81, 369-377.

11. C. J. Hogan and J. Fernandez de la Mora, Journal of the American Society for Mass Spectrometry, 2011, 22, 158-172.

12. S. J. Allen, A. M. Schwartz and M. F. Bush, Analytical Chemistry, 2013, 85, 12055-12061.

13. L. Konermann, E. Ahadi, A. D. Rodriguez and S. Vahidi, Analytical Chemistry, 2013, 85, 2-9.

14. L. Konermann, A. D. Rodriguez and J. Liu, Analytical Chemistry, 2012, 84, 6798-6804.

15. X. Yue, S. Vahidi and L. Konermann, Journal of the American Society for Mass Spectrometry, 2014, 25, 1322-1331.

16. J. B. Fenn, J. Rosell and C. K. Meng, Journal of the American Society for Mass Spectrometry, 1997, 8, 1147-1157.

17. M. Labowsky, J. B. Fenn and J. Fernandez de la Mora, Anal Chim Acta, 2000, 406, 105-118.

18. C. J. Hogan and J. Fernandez de la Mora, Phys Chem Chem Phys, 2009, 11, 8079-8090.

19. I. Romero-Sanz, R. Bocanegra, J. Fernandez de la Mora and M. Gamero-Castano, J Appl Phys, 2003, 94, 3599-3605.

20. V. Znamenskiy, I. Marginean and A. Vertes, J Phys Chem A, 2003, 107, 7406-7412.

21. J. K. Chung and S. Consta, Journal of Physical Chemistry B, 2012, 116, 5777-5785.

22. S. Consta and A. Malevanets, Journal of Chemical Physics, 2013, 138.

23. K. Ichiki and S. Consta, Journal of Physical Chemistry B, 2006, 110, 19168-19175.

24. E. Ahadi and L. Konermann, Journal of the American Chemical Society, 2011, 133, 9354-9363.

25. E. Ahadi and L. Konermann, Journal of Physical Chemistry B, 2012, 116, 104-112.

26. L. Konermann, R. G. McAllister and H. Metwally, Journal of Physical Chemistry B, 2014, 118, 12025-12033.

27. N. A. Fuchs and A. G. Sutugin, Highly dispersed aerosols, Ann Arbor Science Publishers, Ann Arbor,, 1970.

28. R. Gopalakrishnan and C. J. Hogan, Aerosol Science and Technology, 2011, 45, 1499-1509.

29. T. Doussineau, M. Santacreu, R. Antoine, P. Dugourd, W. Zhang, I. Chaduc, M. Lansalot, F. D'Agosto and B. Charleux, Chemphyschem, 2013, 14, 603-609.

30. C. J. Hogan, P. Biswas and D. R. Chen, The Journal of Physical Chemistry B, 2009, 113, 970-976.

31. T. Seto, T. Maekawa, S. Osone, K. Kawamura, T. Yamauchi and Y. Otani, Chem. Eng. Sci., 2013, 85, 46-49.

32. J. Fernandez de la Mora and I. G. Loscertales, J. Fluid Mech., 1994, 260, 155-184.

33. J. Fernandez de la Mora, J Colloid Interf Sci, 1996, 178, 209-218.

34. J. Fernandez de la Mora, L. de Juan, T. Eichler and J. Rosell, Trac-Trend Anal Chem, 1998, 17, 328-339.

35. E. O. Knutson and K. T. Whitby, Journal of Aerosol Science, 1975, 6, 443-451.

36. W. Winklmayr, G. P. Reischl, A. O. Lindner and A. Berner, Journal of Aerosol Science, 1991, 22, 289-296.

37. R. Gopalakrishnan, M. R. Meredith, C. Larriba-Andaluz and C. J. Hogan, Journal of Aerosol Science, 2013, 63, 126-145.

38. N. A. Fuchs, Geofis. Pura Appl., 1963, 51, 185-193.

39. G. P. Reischl, J. M. Mäkelä, R. Karch and J. Necid, Journal of Aerosol Science, 1996, 27, 931949.

40. S. C. Wang and R. C. Flagan, Aerosol Science and Technology, 1990, 13, 230-240. 
41. H. J. C. Berendsen, J. R. Grigera and T. P. Straatsma, Journal of Physical Chemistry, 1987, 91, 6269-6271.

42. A. K. Arumugham-Achari, J. Grifoll and J. Rosell-Llompart, Journal of Aerosol Science, 2013, 65, 121-133.

43. T. Seto, K. Okuyama, L. deJuan and J. Fernandez de la Mora, Journal of Chemical Physics, 1997 , 107, 1576-1585.

44. E. W. McDaniel and E. A. Mason, The Mobility and Diffusion of Ions in Gases 1973.

45. J. Fernández-García and J. Fernández de la Mora, Journal of the American Society for Mass Spectrometry, 2013, 24, 1872-1889.

46. C. Larriba, C. J. Hogan, M. Attoui, R. Borrajo, J. Fernandez-Garcia and J. Fernandez de la Mora, Aerosol Science and Technology, 2011, 45, 453-467.

47. C. N. Davies, Proceedings of the Physical Society, 1945, 57, 259-270.

48. R. A. Millikan, Physical Review, 1923, 22, 1-23.

49. A. Kumar, S. Kang, C. Larriba-Andaluz, H. Ouyang, C. J. Hogan and R. M. Sankaran, Nanotechnology, 2014, 25, 385601.

50. C. Larriba and C. J. Hogan, The Journal of Physical Chemistry A, 2013, 117, 3887-3901.

51. H. Ouyang, C. Larriba-Andaluz, D. R. Oberreit and C. J. Hogan, Journal of the American Society for Mass Spectrometry, 2013, 24, 1833-1847.

52. J. Fernández-García and J. Fernández de la Mora, Phys Chem Chem Phys, 2014, 16, 20500-20513.

53. H. Tammet, Journal of Aerosol Science, 1995, 26, 459-475.

54. A. Wiedensohler, Journal of Aerosol Science, 1988, 19, 387-389.

55. M. P. Allen and D. J. Tildesley, Computer Simulation of Liquids, Oxford Science Publications 1987.

56. R. Gopalakrishnan, T. Thajudeen and C. J. Hogan, Journal of Chemical Physics, 2011, 135, 054302 .

57. H. Ouyang, R. Gopalakrishnan and C. J. Hogan, The Journal of Chemical Physics, 2012, 137, 064316.

58. B. E. Dahneke, in Theory of Dispersed Multiphase Flow, ed. R. E. Meyer, Academic Press, New York1983.

59. D. C. Sahni, Journal of Nuclear Energy, 1966, 20, 915.

60. W. Li and E. J. Davis, Aerosol Science and Technology, 1996, 25, 11-21.

61. M. Gamero-Castano and J. Fernandez de la Mora, Journal of Chemical Physics, 2002, 117, 33453353.

62. S. Chapman and T. G. Cowling, The Mathematical Theory of Non-uniform Gases, Cambridge University Press, Cambridge, 1970.

63. B. E. Poling, J. M. Prausnitz and J. P. O'Conell, The Properties of Gases and Liquids, McGraw Hill Inc.2001.

64. M. H. Factorovich, V. Molinero and D. A. Scherlis, Journal of the American Chemical Society, 2014, 136, 4508-4514.

65. S. M. Thompson, K. E. Gubbins, J. P. R. B. Walton, R. A. R. Chantry and J. S. Rowlinson, The Journal of Chemical Physics, 1984, 81, 530-542.

66. C. S. Dutcher, X. L. Ge, A. S. Wexer and S. L. Clegg, J. Phys. Chem. C, 2011, 115, 16474-16487. 